\title{
Unternehmen Europa
}

\author{
Udo Krolzik
}

Das Zusammenwachsen in Europa wird den Wettbewerb um den besten Weg bei der Lösung bestimmter sozialer Probleme fördern. Die freigemeinnützigen Unternehmen in Deutschland müssen dazu ihre Zurückhaltung gegenüber Europa und dem Markt überwinden und neue Wege bei der Erbringung sozialer Dienstleistungen einschlagen.

Tief verwurzelte Vorurteile fordern eine Rechtfertigung für ein Engagement deutscher Sozialunternehmen in Europa. Noch immer scheint es ungewöhnlich, dass sich ein freigemeinnütziger Träger europäisch engagiert! Dieser Eindruck stimmt mit der Realität überein: Sieht man einmal von »Hilfstransporten « und Unterstützungsleistungen $a b$, so sind nur wenige freigemeinnützige Sozialunternehmen in der Europäischen Union tätig geworden!

Grund dafür war, dass freigemeinnützige deutsche Unternehmen sich am Sozialstaat orientiert und an ihn gebunden haben. Seit Beginn des 20. Jahrhunderts haben sich die wohlfahrtsverbandlich organisierten sozialen Dienstleister an die staatlichen Umverteilungsströme angedockt und haben so immer eher als Verteiler und Verwalter der staatlichen Sozialleistungen gehandelt und nicht als Sozialunternehmen. So ausgerichtete freigemeinnützige Leistungserbringer hatten und zum großen Teil haben den europäischen Binnenmarkt nicht im Blick.

Verstärkt wurde diese Interesselosigkeit deutscher freigemeinnütziger Unternehmen durch die Tatsache, dass sie in anderen europäischen Staaten völlig anderen Sozialsystemen als das auf dem bismarckschen System aufbauenden deutschen begegnen - sieht man von Parallelen in Österreich und den Beneluxländern und in begrenzterem Umfang in Frankreich einmal ab. Auch die osteuropäischen Beitrittsländer habe nach der Öffnung zum Westen zwar geschwankt, ob sie das deutsche System übernehmen sollen, es dann aber doch weitgehend gelassen.

Da die Wohlfahrtspflege als verwaltende und nicht als unternehmerische erlebt wurde, hat man ihr auch kein europäisches Engagement zugetraut. Im Jahre 1991 stellte eine Studie der Prognos AG fest, dass Europa für die Wohlfahrtsverbände noch kein Thema ist. Auch heute scheinen sich Freigemeinnützige immer noch rechtfertigen müssen, wenn sie sich in Europa engagieren.

Spätestens seit Mitte der 1990er Jahre sind politisch gewollt die sozialstaatlichen Voraussetzungen in Deutschland abgebaut und der Wettbewerb bei sozialen Dienstleistungen aufgebaut worden. Die damit einsetzende Entwicklung zu einer Sozialwirtschaft in Deutschland

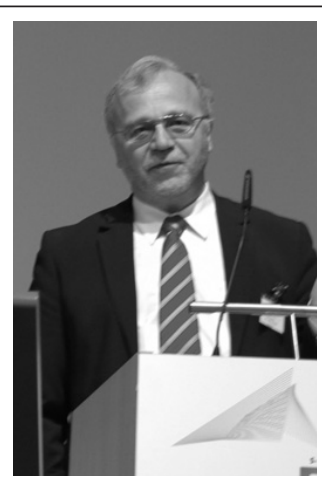

Dr. Udo Krolzik (59) ist Vorsitzender des Vorstandes des Evangelischen Johanneswerkes e. V. in Bielefeld. Er hat Betriebswirtschaft und Theologie in Hamburg und Edinburgh studiert und war wissenschaftlicher Assistent am kirchen- und dogmengeschichtlichen Seminar der Universität Hamburg. Von 1989 bis 1993 war er Pastor in Hamburg-Hummelsbüttel, dann wurde er theologisches Mitglied im Vorstand des Evangelischen Johanneswerkes, dessen Vorsitz er 1996 übernahm. E-Mail udo-krolzik@johanneswerk.de

wurde verstärkt durch die gleichzeitige europäische Entwicklung zu einem wachsenden Binnenmarkt, der immer stärker auch die nationalstaatlichen Sozialmärkte erfasste.

\section{Drei Vorurteile gegen Europa}

Die freigemeinnützigen Sozialunternehmen haben sich nur zögernd für ein europäisches Engagement geöffnet! Lange Zeit hat auch die Europäische Kommission die deutschen Wohlfahrtsverbände mit ihren 1,3 Millionen Mitarbeitenden und einem Wirtschaftsvolumen von 60 Milliarden Euro nicht wahrgenommen.

Drei tief verwurzelte Vorurteile, die von vielen Verantwortlichen bei den freigemeinnützigen Unternehmen geteilt werden, schätzen diese Unternehmen als europauntauglich ein.

- Das erste Vorurteil besagt, Europa betreffe die freigemeinnützigen Unternehmen nicht. Viele Verantwortliche in der Wohlfahrtspflege und Politik waren und sind dieser Auffassung: »Die Europäische Union ist eine Wirtschafts- und keine Sozialunion.« Dieser Satz ist in Politik und Wohlfahrtsverbänden zu einem Ohrwurm geworden, der das Schlaflied hervorbrachte: Alles bleibt beim Alten, ihr braucht euch nicht zu kümmern! Die Verträge von Maastricht und besonders Amsterdam und die Lissabonner Strategie haben deutlich gemacht, dass der Europäische Rat zusammen mit dem Europäischen Parlament auch in der Sozialpolitik handeln kann. In Anwendung der Verträge tendiert die Rechtsprechung 


\section{Europa sozial managen}

Das fünfte Branchentreffen der Sozialwirtschaft war auch das größte: Über 300 Führungskräfte aus Verbänden und Unternehmen trafen sich am 26. und 27. April 2007 in Magdeburg zum Meinungsaustausch. Im Mittelpunkt der Vorträge und Diskussionen stand das Thema Europa.

»Wir brauchen einen Wettbewerb«, gab Dr. Peter Neher, Präsident der Bundesarbeitsgemeinschaft der Freien Wohlfahrtspflege und Präsident des Deutschen Caritasverbandes, in seiner Begrüßungsansprache als Tenor vor und fügte hinzu: »Aber einen Wettbewerb um Qualität und nicht um den geringsten Preis auf Kosten der Klienten und der Mitarbeitenden.«

Die Sozialwirtschaft in Deutschland lasse sich längst nicht mehr nur in nationalen Grenzen denken, so Neher. Europa mit den Rahmenbedingungen der Europäischen Union sowie der europäische Wettbewerb seien für die Unternehmen eine Realität. Das gleiche gelte für den deutschen Sozialstaat. Er müsse sich an den anderen Sozialstaatsmodellen in Europa messen lassen. Gleichzeitig bräuchten wir einen Prozess zur Verständigung über gemeinsame Standards auf europäischer Ebene. Auch der Wettbewerb sozialer Dienstleister sei im europarechtlichen Kontext zu gestalten. Ebenso stünden die sozialen Berufe unter der Herausforderung der Mobilität in der Europäischen Union. Neher: »Diese vielen Dimensionen will der Kongress unter den Stichworten >Werte, Wettbewerb, Finanzen ausleuchten.«

Veranstalter dieses alle zwei Jahre stattfindenden Branchentreffs sind die Bundesarbeitsgemeinschaft der Freien Wohlfahrtspflege, die Bank für Sozialwirtschaft und die Nomos Verlagsgesellschaft. Die Schirmherrschaft übernommen hatte Dr. Ursula von der Leyen, Bundesministerin für Familie, Senioren, Frauen und Jugend. Der Kongress der Sozialwirtschaft war Teil der offiziellen Veranstaltungen der deutschen Ratspräsidentschaft der Europäischen Gemeinschaft im ersten Halbjahr 2007.

Die Sozialwirtschaft ist eine der größten Wirtschaftszweige der Bundesrepublik. Allein in den Betrieben der sechs anerkannten Wohlfahrtsverbände - Arbeiterwohlfahrt, Caritas, Deutsches Rotes Kreuz, Diakonisches Werk, Paritätischer Wohlfahrtsverband und Zentralwohlfahrtsstelle der Juden - arbeiten hauptberuflich rund eineinhalb Millionen Menschen, das sind knapp vier Prozent aller Erwerbstätigen in Deutschland. Zusätzlich engagieren sich noch zweieinhalb bis drei Millionen Bürgerinnen und Bürger ehrenamtlich als Hospizhelfer, gesetzliche Betreuer oder als persönlich haftende Vereinsvorstände.

Zu dem Kongress waren über 300 Führungskräfte aus gewerblichen, gemeinnützigen und kommunalen Unternehmen der Sozialwirtschaft gekommen. In zwei Plenumsveranstaltungen und sechs Foren diskutieren sie mit Referenten aus ganz Europa die konkreten Auswirkungen der europäischen Einigung auf die Sozialwirtschaft. Untersucht wurden beispielsweise die Vor- und Nachteile der verschiedenen Sozialstaatsmodelle in der Europäischen Union, die Folgen des europäischen Vergaberechts auf deutsche Leistungserbringer, die Anpassungsnotwendigkeiten von Organisationsstrukturen und Rechtsformen angesichts des europäischen Wettbewerbsrechts, die Aus-, Fort- und Weiterbildung sozialer Berufe angesichts steigender Mobilität in Europa, die Frage nach gemeinsamen Werten sowie die Auswirkungen der Dienstleistungsrichtlinie der Europäischen Union für den Import und den Export sozialer Dienstleistungen.

In einer Ausstellung präsentieren sich während des gesamten Kongresses einzelne Wohlfahrtsverbände, soziale Einrichtungen, Weiterbildungsstätten, Verlage, Finanzdienstleister und Wirtschaftsprüfgesellschaften mit ihren fachlichen Konzepten, speziellen Dienstleistungen und sonstigen Angeboten. Bei einem Empfang am Abend des ersten Veranstaltungstages verlieh die Bank für Sozialwirtschaft die Preise der von ihr ausgelobten »Sozialkampagne « für innovative und aufmerksamkeitsstarke Werbekampagnen zu sozialen Themen.

Die Vorträge, Referate und Präsentationen des Treffens werden als Sammelband unter der Herausgeberschaft der Bundesarbeitsgemeinschaft der Freien Wohlfahrtspflege in der Nomos Verlagsgesellschaft veröffentlicht. Der nächste Kongress ist für das Jahr 2009 geplant.

Kongress der Sozialwirtschaft, Oppelner Straße 130, 53119 Bonn, Telefon 0228 6685-0, Fax 0228 6685-211, E-Mail sozkon@awobu.awo.org, Internet http://www.kongress-der-sozialwirtschaft.de

des Europäischen Gerichtshofes dazu, auch bei den sozialen Dienstleistungen die Anwendung des EG-Rechtes in Bezug auf den Binnenmarkt zu prüfen.

- Das zweite Vorurteil war und ist es teilweise immer noch: Europa ist noch weit weg! Bis Europa bei uns in Deutschland und besonders in den Wohlfahrtsverbänden ankommt, wird noch viel Zeit vergehen! Tatsächlich hat das Zusammenwachsen Europas in den letzten 15 Jahren enorm an Fahrt gewonnen. Politisch ist dies im Fall des Eisernen Vorhangs begründet, technologisch im weltumspannenden Datennetz und gesellschaftlich in der gesamteuropäischen Säkularisierung. Gemeinsame gesellschaftliche Herausforderungen führen dazu, dass auch im sozialpolitischen Bereich die Geschwindigkeit des Zusammenwachsens nicht nachlassen, sondern sich noch erhöhen wird: eine ungünstige demografische Entwicklung und hohe Arbeitslosigkeit. Es liegt nahe, darauf gemeinsam zu antworten und für die damit verbundene soziale Ausgrenzung und Benachteiligung gemeinsame Strategien zu entwickeln.

- Das dritte Vorurteil ist, die freigemeinnützigen Unternehmen können zu einem zusammenwachsenden Europa keinen Beitrag leisten - und seien dafür auch nicht zuständig. Häufig werde ich gefragt, nicht nur von Verantwortlichen in den Wohlfahrtsverbänden, sondern auch von Politikern, was macht ihr vom Evangelischen 
Johanneswerk im fernen Spanien? Der darin enthaltene Vorwurf ist entweder, warum geht ihr in die Ferne, statt euch an eurer Lebenswelt zu orientieren. Aber genauso wie es keinen Grund gab, in Bielefeld zu bleiben und auch nicht in Westfalen oder Nordrhein-Westfalen, gibt es keinen Grund sich auf Deutschland zu beschränken. Ein weiterer Vorwurf schwingt in der Frage »Was macht ihr in Spanien? « mit: Dort sind doch nur die Reichen. Als freigemeinnützige Unternehmen wenden wir uns nicht nur den Einkommensschwachen zu, sondern allen Menschen, die unsere Dienstleistungen brauchen. In einem zusammenwachsenden Europa können und wollen wir uns nicht den Blick verengen lassen auf die Einkommensschwachen in unserem Land, sondern unsere Gemeinwohlorientierung nimmt auch das Wohl der Menschen in anderen Ländern in den Blick und nicht nur das der Einkommensschwachen, sondern auch der Kranken oder sonst der Dienstleistung Bedürftigen.

Freigemeinnützige Sozialunternehmen sind keineswegs europauntauglich, sondern taugen aufgrund ihrer Gemeinwohlorientierung für den Auftrag der Europäischen Union gegen Ausgrenzung und Benachteiligung und für sozialen Zusammenhalt und Teilhabe zu wirken. Insofern sind die Freigemeinnützigen Im Hinblick auf Europa besonders zukunftsfähig. Sie sind dies nicht aufgrund ihrer Gemeinnützigkeit, sondern weil sie am Gemeinwohl orientiert sind. Dies führt in Deutschland zur Gemeinnützigkeit im Sinne der Abgabenordnung, muss es aber nicht zwingend in anderen europäischen Ländern. Gemeinnützigkeit ist keine conditio sine qua non für eine Engagement in Europa.

\section{Perspektive Europa}

Freigemeinnützige haben nur eine europäische Zukunft oder sie haben keine Zukunft. Freigemeinnützige Unternehmen sind aber nicht nur europatauglich, sondern sie haben auch nur eine Zukunft, wenn sie sich gezielt europäisieren. Europa, das war, wie Konrad Adenauer einmal gesagt hat, zunächst nur ein Traum für wenige, später eine Hoffnung für viele, und ist heute eine Notwendigkeit für alle geworden. Das gilt auch für die freigemeinnützigen Unternehmen. Sie müssen sich auf eine europäische Zukunft ausrichten oder sie haben keine Zukunft.

Rund zwei Drittel aller nationalen Gesetze sind durch Europa bestimmt. Und auch das 2005 verabschiedet Maßnahmenpaket der Europäischen Kommission zur Finanzierung von gemeinwirtschaftlichen Leistungen zeigt, wie weit gerade soziale Dienstleistungsangebote von Europa bestimmt sind. Das Maßnahmenpaket stellt zwar fest, dass unsere Angebote in Deutschland im Wesentlichen nicht unter das Beihilfeverbot fallen, aber gerade dadurch, dass die Kommission entscheidet, was unter diese Vorgaben fällt, macht deutlich, wie umfassend wir in unserem Handeln von europäischen Entwicklungen und Entscheidungen abhängen.

Es mag so scheinen, dass es mit dem Scheitern des EUVerfassungsentwurfes gelungen ist, die wachsende $\mathrm{Zu}$ ständigkeit der EU-Organe für nationale Politiken abzuwehren. Tatsächlich zeigt es aber nur eins: Wie in ihren Anfängen sind es nicht politische Einsicht und politi- scher Wille, die die europäische Gemeinschaft formen, sondern wirtschaftliche Überzeugungen und Notwendigkeiten trieben die europäische Einigung voran und gaben ihr Gestalt. So war schon 1954 durch die französische Nationalversammlung der Plan für eine Europäische Verteidigungsgemeinschaft gescheitert. Damit war allen weiteren Plänen zur Gründung einer umfassenden Europäischen Politischen Gemeinschaft der Boden entzogen. Die EWG entsprang dann der Logik und den Kräften der älteren wirtschaftlichen Europäischen Gemeinschaften für Kohle und Stahl. Die wirtschaftliche Logik und ihre Kräfte blieben auch in der Folge bestimmend - formten und trieben den Prozess der europäischen Gemeinschaftsbildung voran. Und auch jetzt nach dem Scheitern der Ratifizierung des Europäischen Verfassungsentwurfes durch die EU-Staaten werden wirtschaftliche Einsichten und Notwendigkeiten die europäische Einigung vorantreiben.

Der europäische Binnenmarkt hat zunehmend die nationale Struktur der Sozialpolitik in Frage gestellt. Es gäbe eine ganze Reihe von Punkten zu benennen, in denen sich die europäische Landschaft schon verändert hat und wovon die sozialen Belange Europas betroffen sind. Ich zähle die wichtigsten auf:

- Euro: Verbunden mit der grundsätzlichen Dienstleistungsfreiheit eröffnet der Euro neue Möglichkeiten, über Grenzen hinweg Preise zu vergleichen, Leistungen anzubieten und wahrzunehmen. Er beschleunigt die Entstehung eines europäischen Dienstleistungsmarktes erheblich, indem er den direkten Preisvergleich in den Staaten der Währungsunion erlaubt, ohne Umrechnung und die damit verbundenen Ungenauigkeiten und ohne Verluste beim Währungstausch. Auch auf die grenzüberschreitende Aufnahme von Arbeitsverhältnissen in Mitgliedstaaten der Währungsunion wird sich der Euro förderlich auswirken. Und den Anbietern erleichtert er es, ihre Leistungen über Grenzen hinweg anzubieten.

\section{- Gemeinnützigkeit, Wettbewerbs- und Beihilfe-} kontrolle: Die Bedeutung der Wohlfahrtsverbände ist auf europäischer Ebene inzwischen erkannt und eine einseitige Bevorzugung des französischen Modells der »économie sociale« abgewendet. So wird in dem Entwurf der europäischen Verfassung in Artikel 34 der Bedeutung der Verbände Rechnung getragen und der offene, transparente und regelmäßige Dialog der Organe der Europäischen Union mit den repräsentativen Verbänden festgeschrieben. Dennoch ist im Zusammenhang mit der Debatte über die Daseinsvorsorge abzusehen, dass es eine Regelung geben wird, die sozialen Dienstleistungen - zumindest teilweise - als wirtschaftliche Leistungen definieren wird. Damit fallen diese Leistungen unter die Wettbewerbskontrolle. Verwoben damit ist die Frage nach der Gemeinnützigkeit oder die Frage nach staatlichen Zuschüssen. Verstoßen diese nicht gegen das Beihilfeverbot? Eine besondere Brisanz erhält die europäische Infragestellung der Gemeinnützigkeit bei uns in Deutschland, weil auf nationaler Ebene der Wettbewerb teilweise bei sozialen Dienstleistungen bewusst intendiert ist. Dies ist beispielsweise eindeutiges Interesse der Pflegeversicherung. Deshalb muss das Thema Wettbewerb für die freigemeinnützigen Unternehmen zunächst national aufgegriffen und geklärt werden. 
- Arbeitsrecht: Ein Beispiel, bei dem die freigemeinnützigen Unternehmen von den europäischen Arbeitsrechtsregelungen betroffen ist, stellt die Bewertung des Bereitschaftsdienstes als Arbeitszeit dar. Ein weiterer kritischer Punkt ist das Diskriminierungsverbot (Art. 13) insbesondere aufgrund der Religionszugehörigkeit. Hier könnte der Tendenzschutz einiger freigemeinnütziger Unternehmen möglicherweise hinterfragt werden.

- Ausschreibungspflicht: Da öffentliche Aufträge ab einer bestimmten Höhe EU-weit ausgeschrieben werden müssen, könnte dies beispielsweise auf den Krankenhausbereich und andere Arbeitsfelder freigemeinnütziger Unternehmen angewendet werden.

- Ethik: Angesichts der grenzüberschreitenden wissenschaftlichen Zusammenarbeit und eines europäischen Wirtschaftsraumes besteht auf europäischer Ebene ein dringendes Bedürfnis nach einheitlichen ethischen Standards - insbesondere in der Bioethik. Ein erster Beleg dafür ist die sogenannte Bioethikkonvention. Deutschland und die freigemeinnützigen Unternehmen werden von solchen ethischen Standards auf europäischer Ebene immer mehr betroffen sein.

- Europäische Konkurrenten: Die Schaffung eines europäischen Binnenmarktes durch die Niederlassungsfreiheit hat dazu geführt, dass die nationalen Märkte von europäischen Anbietern durchdrungen werden und sich ein europäischer Markt gebildet hat! Beispiele: der französische Konzern LVL und schwedische Krankenhausträger. Die Hamburger Endoklinik hat eine orthopädische Abteilung in einem dänischen Krankenhaus eröffnet. Seit dem 1. Dezember 2003 können dänische Patienten von Ärzten der Hamburger Klinik in Dänemark behandelt werden. Außerdem können bestimmte Gesundheitsleistungen im Ausland wahrgenommen werden, wie etwa Kuren in Ungarn, der Tschechischen Republik, Polen. In den nahezu 30 »Euregios« funktioniert die Zusammenarbeit gerade im Bereich der grenzüberschreitenden Gesundheitsdienstleistungen. So können sich in der Euregio Maas-Rhein niederländische, deutsche und belgische Patienten seit 1997 grenzüberschreitend von Fachärzten behandeln lassen. Schon seit Anfang der 1990er-Jahre arbeiten mehrere ostdeutsche Grenzregionen zusammen, so die Region Pomerania, wo deutsche, polnische und schwedische Grenzgebiete bei der Suchtvorbeugung und beim Aufbau eines telemedizinischen Netzes kooperieren. Die Arbeitnehmerfreizügigkeit führt dazu, dass immer mehr Mitarbeitende in Sozialunternehmen aus anderen europäischen Ländern tätig sind. Im Evangelischen Johanneswerk haben wir über 320 Mitarbeitende von 6.300, die keine deutsche Staatsangehörigkeit besitzen, aus 48 Staaten. Nicht erfasst sind dabei die Mitarbeitenden, die zwar aus Polen, Rumänien, Ungarn und Russland gekommen sind, aber die deutsche Staatszugehörigkeit haben.

- Fördergelder: In erheblichem Umfang werden von der Europäischen Union Gelder zur Förderung der sozialen Entwicklung bereitgestellt. Diese europäischen Strukturfondmittel werden in Deutschland über Bund und Länder verteilt. Besondere Berücksichtigung finden dabei Projekte zur Gesundheitsförderung, Beseitigung von Arbeitslosigkeit und sozialer Ausgrenzung. Zusätzlich werden von der Union umfangreiche Mittel zur
Entwicklung der neuen Technologien bereitgestellt. Dabei werden besonders mit dem 7 . Rahmenprogramm 2007 Projekte gefördert, die allen Gruppen Zugänge zu diesen Technologien ermöglichen, indem sie Bedienungen entwickeln, die niemanden ausschließen.

Euro, Wettbewerbs- und Beihilfekontrolle, Arbeitsrecht, Ausschreibungspflicht, europäische Konkurrenz, Fördergelder betreffen auch die freigemeinnützigen Sozialunternehmen. Anbieter von personennahen Dienstleistungen machen immer häufiger grenzüberschreitende Angebote in der Europäischen Union. Menschenbild und Leitbilder freigemeinnütziger Unternehmen und die darauf fußenden ethischen Urteile werden zunehmend von der europäischen Diskussion beeinflusst.

Deshalb gilt heute: Freigemeinnützige Unternehmen haben nur eine europäische Zukunft oder sie haben keine Zukunft. Europa ist zur Notwendigkeit für freigemeinnützige Unternehmen geworden.

\section{Chance Europa}

Ein Engagement in Europa ist für freigemeinnützige Unternehmen nicht nur eine Notwendigkeit, sondern auch eine große Chance:

- Leistungserbringung im europäischen Ausland: Genauso wie freigemeinnützige Unternehmen ihre Leistung über Grenzen der Kommunen und Bundesländer hinweg anbieten, werden sie das über nationale Grenzen hinweg tun. Teilweise geschieht das schon. Denn für eine ausschließliche Antwort auf Bedürfnisse von Menschen in einem engen geographischen und politischen Raum gab es weder im 19. Jahrhundert bei der Entstehung der freigemeinnützigen Unternehmen noch heute vernünftige oder gar gemeinnützige Gründe. Europaweit wachsen die Märkte für soziale Dienstleistungen, insbesondere der Pflegemarkt. In einigen Ländern steht der Aufbau von Versorgungsstrukturen noch am Anfang und kann von den Ländern aufgrund der rasanten Entwicklung nicht alleine bewältigt werden. Hierzu werden sich transnationale Kooperationen bilden. Solche Kooperationen erschließen Zugänge zu europäischen Märkten und erlauben Angebote zu machen, die unterschiedlichen Nachfragegruppen gerecht werden. So können etwa deutsche Spezialkliniken mit Allgemeinkrankenhäusern in anderen Ländern kooperieren und dort ihre besondere Kompetenz auf einem Fachgebiet anbieten. Ähnliche Vorhaben äußerten Vertreter aus Dänemark, Großbritannien und Holland bezogen auf den deutschen Markt. So bewirkt die Europäische Union langfristig das Entstehen transnationaler Netzstrukturen im Bereich der sozialen Sicherheit.

- Werben von Mitarbeitern im europäischen Ausland: Schon heute sind in freigemeinnützigen Einrichtungen nicht nur Arbeitskräfte aus der jeweiligen Kommune oder dem jeweiligen Bundesland oder überhaupt aus Deutschland tätig. Dies gilt in besonderem Maße für die Altenhilfe, in der die Anzahl, der aus Osteuropa stammenden Mitarbeiter stetig zunimmt. Darüber hinaus gibt es seit den 1980er-Jahren Mitarbeitende aus anderen europäischen und überseeischen Ländern. Der akute Pflege- 
kräftemangel wie die Freizügigkeit der Arbeitnehmer in der Union wird besonders durch die Osterweiterung zu einer Europäisierung der Mitarbeiterschaft führen. Diese Möglichkeit muss bewusst gestaltet werden.

- Austausch von Ideen und Mitarbeitenden: Die europäischen Länder stehen in vielen Bereichen vor gleichen Herausforderungen, so bei der Beschäftigungspolitik, der Armutsbekämpfung, der demografischen Entwicklung. Durch Austausch der Ideen und der Erfahrungen, die mit unterschiedlichen Wegen gemacht wurden, sowie von Mitarbeitenden können die Länder viel von einander lernen. Ein solcher Austausch geschieht beispielsweise in den europäischen Netzwerken für Demenzarbeit oder Behindertenarbeit. Lernen ließe sich in der Pflegeausbildung oder bei der Gewinnung Ehrenamtlicher, wie sie etwa in Großbritannien durch »Business in the community « geschieht. Es wird dann nicht darum gehen, dass die Sozialsysteme harmonisiert oder zusammengeführt werden, sondern der Wettbewerb der besten Wege und der besten Praxis formen das neue Leitbild einer europäischen Angebotsstruktur. Neben dem Austausch von Ideen und Erfahrungen könnte eine im europäischen Horizont handelnde Sozialwirtschaft den Austausch von Mitarbeitenden fördern. Damit würde sowohl eine fachliche als auch eine allgemein menschliche Horizonterweiterung angeregt und ermöglicht werden. So würde ein Beitrag zur Lösung von sozialen Problemen in europäischen Regionen geleistet werden, der über die Hilfstransporte hinausginge.

- Orientierung am Gemeinwohl: Die Besonderheit gemeinwohlorientierter Dienstleistungen mit ihrer ethischen und spirituellen Dimension und Verpflichtung muss in die europäische Entwicklung eingebracht werden und es muss darauf geachtet werden, dass die europäische Rechtsentwicklung die Handlungsspielräume der Freigemeinnützigen nicht einschränkt. Bei der Mitgestaltung Europas können die Wohlfahrtsverbände ihre Rolle als Element und Komponente der Zivilgesellschaft in Erinnerung rufen. Gerade auf dem Hintergrund der immer wieder vermissten Bürgernähe Brüssels ist diese Rolle für die europäische Entwicklung von großer Bedeutung.

- Lernen für multikulturelle Pflege: Ein wichtiger Lernschritt in einem zusammenwachsenden Europa wird eine multikulturelle Pflege sein. In Zukunft werden aufgrund der Arbeits-, Gesundheits- oder Wohlstandsmigration immer häufiger Menschen in einem fremden kulturellen Umfeld von Angehörigen anderer Kulturen gepflegt werden. Bisher ist die Pflege in diesem Setting der diakonischen Findigkeit und Genialität einzelner freigemeinnütziger Unternehmen überlassen. Hier könnten gezieltere Untersuchungen und darauf aufbauende Angebote gemacht werden, damit es zu einer am Gemeinwohl motivierten Fachlichkeit kommt.

- Ausbildung: Europa bietet Ausbildungsträgern der Wohlfahrtspflege die Möglichkeit, ihre Ausbildung insbesondere in den Pflegeberufen auf europäischer Ebene anzugleichen. Wir brauchen dringend eine mehrstufige europäische Pflegeausbildung. Dabei wird es einerseits um die Akademisierung der Pflege in bestimmten Bereichen gehen. Andererseits ist aber auch dringend eine Kurzausbildung für bestimmte Tätigkeiten in der Pflege gefordert.
Thesen: Warum wir uns in Europa engagieren müssen

Die Gründe freigemeinnütziger Unternehmen für ein Engagement in Europa lassen sich in vier Thesen zusammenfassen.

- Freigemeinnützige Unternehmen machen ihre Angebote dort, wo die Menschen sind, die sie brauchen. Und diese Menschen sind zunehmend auch in anderen europäischen Staaten, etwa an den Küsten Spaniens. So wurden wir von den deutschen evangelischen Auslandsgemeinden gebeten für die dort ansässigen Senioren ein Versorgungsangebot zu machen. Ebenso bat uns die Deutsche Botschaft in Madrid um solche Angebote für die geschätzten 700.000 Deutschen in Spanien und zusätzlich Sozialberatung anzubieten. In Griechenland riefen uns die deutschsprachigen Gemeinden auf, sie bei der Versorgung der deutschen Witwen zu unterstützen, da diese aus den griechischen sozialen Versorgungsnetzen herausfallen und in der Regel nicht nach Deutschland zurückkehren können und auch nicht wollen.

- Wir engagieren uns in Europa, weil wir fachlich gut sind! So hat eine Untersuchung der fachlichen Qualität, die kürzlich in der autonomen Region Valencia stattfand, festgestellt, dass unsere beiden Residenzen als einzige fachlich gute Angebote machen. Darüber hinaus haben wir in multikultureller Versorgung Erfahrung und lernen ständig hinzu. In unseren spanischen Häusern leben acht bis zwölf Nationen unter einem Dach. Unsere Mitarbeitende müssen nicht nur drei Sprachen sprechen, sondern wir haben auch Personal anderer Nationalitäten - nicht nur spanische und deutsche - angestellt. Dabei geht es darum, Pflegestandards zu entwickeln, die diese Multikulturalität berücksichtigen. Darüber hinaus haben wir »Sonderentwicklungen« wie die Rehabilitation, die wir ergänzend einbringen können.

- Zum dritten engagieren wir uns in Europa, weil wir andere Länder bei dem Aufbau von Versorgungsstrukturen unterstützen können. In zahlreichen europäischen Gesellschaften haben in den letzten Jahren Entwicklungen eingesetzt, die bei uns in den 1980er Jahren stattfanden und auf die wir mit entsprechenden Versorgungsnageboten reagiert haben. Heute können wir mit unseren Erfahrungen andere Länder unterstützen. So haben wir in verschiedenen Ministerien in Madrid unsere Vorstellungen vorgetragen. Dabei wird zunehmend deutlich, dass wir nicht unsere Versorgungsstrukturen der 1980er und 1990er Jahre anbieten sollen, sondern die der Gegenwart.

- Die freigemeinnützigen Unternehmen können mit ihrer Gemeinwohlorientierung und ihrem Menschenbild ein entstehendes europäisches Sozialmodell mit gestalten. Es ist Auftrag und Ziel vieler europäischer Institutionen den sozialen Zusammenhalt und die Teilhabe in Europa zu fördern und zu stärken. Dazu können wir Wesentliches beitragen.

Gründe genug, um sich in Europa als freigemeinnütziges Unternehmen zu engagieren! 